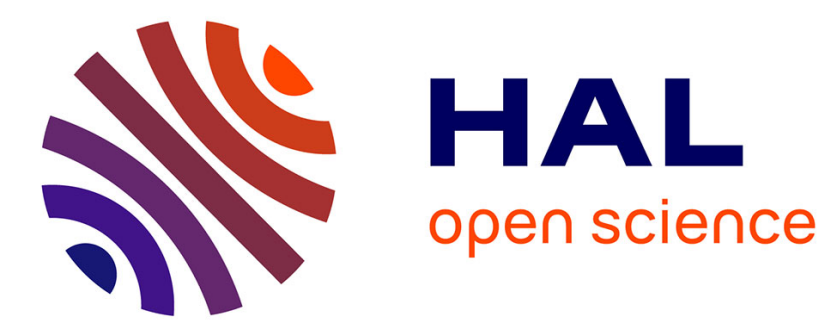

\title{
Cooperative Resynchronization to Improve the Reliability of Colocated IEEE 802.15.4-TSCH Networks in Dense Deployments
}

\author{
Sahar Ben Yaala, Fabrice Theoleyre, Ridha Bouallegue
}

\section{- To cite this version:}

Sahar Ben Yaala, Fabrice Theoleyre, Ridha Bouallegue. Cooperative Resynchronization to Improve the Reliability of Colocated IEEE 802.15.4-TSCH Networks in Dense Deployments. Ad Hoc Networks, 2017, 64, pp.112-126. 10.1016/j.adhoc.2017.07.002 . hal-02565964

\author{
HAL Id: hal-02565964 \\ https://hal.science/hal-02565964
}

Submitted on 6 May 2020

HAL is a multi-disciplinary open access archive for the deposit and dissemination of scientific research documents, whether they are published or not. The documents may come from teaching and research institutions in France or abroad, or from public or private research centers.
L'archive ouverte pluridisciplinaire HAL, est destinée au dépôt et à la diffusion de documents scientifiques de niveau recherche, publiés ou non, émanant des établissements d'enseignement et de recherche français ou étrangers, des laboratoires publics ou privés. 
(c) 2017

This work is licensed under a Creative Commons "Attribution-NonCommercialNoDerivatives 4.0 International" license.

(9) $\Theta \Theta$

\title{
Cooperative Resynchronization to Improve the Reliability of Colocated IEEE 802.15.4-TSCH Networks in Dense Deployments
}

\author{
Sahar Ben Yaala ${ }^{\mathrm{a}, *}$, Fabrice Théoleyre ${ }^{\mathrm{b}, *}$, Ridha Bouallegue ${ }^{\mathrm{a}}$ \\ ${ }^{a}$ Innov'COM Laboratory, Sup'Com, University of Carthage, Tunisia \\ ${ }^{b}$ ICube Laboratory, CNRS / University of Strasbourg, Boulevard Sebastien Brant, 67412 Illkirch Cedex, France
}

\begin{abstract}
With the large adoption of small and smart objects, the interest of researchers to define new protocols to meet Internet of Things (IoT) requirements is growing. In particular, the Industrial Internet of Things (IIoT) aims to provide high reliability and upper bounded end-to-end latency while interconnecting a large collection of sensors and actuators. The TimeSlotted Channel Hopping (TSCH) mode of IEEE 802.15.4 exploits a slow channel hopping approach to combat efficiently external interference (e.g. Wi-Fi, Bluetooth). We envision in the future very dense deployments and we investigate here the mutual influence among different colocated TSCH networks. We first study analytically with a simple model the performance of several TSCH networks, able or not to be synchronized with each other. We emphasize the multiplication of collisions, exacerbated by clock drifts when no cooperation is achieved. We also highlight experimentally that a mutual synchronization improves very significantly the reliability. We propose here a mechanism to re-synchronize distributively the border routers of different TSCH networks. Our thorough experimental evaluation illustrates the relevance of our approach, able to guarantee high-reliability, even in very dense topologies.
\end{abstract}

Keywords: IEEE 802.15.4-TSCH; colocated networks; dense deployments; distributed synchronization; border routers; collision avoidance; experimental evaluation

\section{Introduction}

Recent advances in technology made possible the creation of smart objects that can be interconnected to create the Internet of Things (IoT). Low power devices create the opportunity to interact with the environment [1]: they measure in real time a collection of physical metrics (e.g. temperature, human 5 presence) or react dynamically by triggering actuators. This Internet of Things is expected to be used in Smart Cities [2], or industrial [3] and healthcare [4] applications.

The Industry 4.0 aims currently to design flexible factories: by integrating low-power radio objects, we can reconfigure easily the manufacturing process and improve the productivity [5. Similarly, healthcare will rely on small embedded devices, able to collect physiological measures in real time [4. However, most of the applications in the Industrial Internet of Things (IIoT) require very low and stable latency and high network reliability [6], and, thus cannot accommodate anymore a best effort approach.

\footnotetext{
${ }^{*}$ Corresponding authors

Email addresses: sahar \underscorechar by\underscorechar ing@live.fr (Sahar Ben Yaala), theoleyre@unistra.fr (Fabrice Théoleyre), ridha.bouallegue@supcom.tn (Ridha Bouallegue)
} 
To reduce the energy consumption, the nodes have to turn their radio off [7]. A lower duty cycle ratio means also a longer node's lifetime: the network is able to operate longer. The MAC protocol is in charge of defining when a node has the right to transmit, so that the receiver is awake and ready to receive the signal correctly. Observing the strict guarantees and requirements of the IIoT lead the researchers to design deterministic algorithms for medium access.

The IEEE 802.15.4-2015 Timeslotted Channel Hopping (TSCH) mode 8 is particularly accurate to address these critical requirements. It supports slow channel hopping to combat external interference: each transmission opportunity uses a different physical channel to avoid repetitive collisions. The channel hopping 20 feature is widely used in industrial wireless networks to provide high reliability, such as ISA100.11a [9] and WirelessHART [10. TSCH supports a global schedule to save energy: when a node is not involved in a transmission or a reception, it just has to turn its radio off. This standard is particularly relevant to provide high reliability and low latency [1].

Smart Cities envision a wide adoption of the Internet of Things to provide new innovant services 2].

${ }_{25}$ For instance, traffic regulation and smart parking 12 rely on a large collection of sensors able to control a set of traffic lights in order to reduce traffic jams. Similarly, vehicular networks now integrate a large collection of devices using different wireless technologies 13. We envision very dense deployments, where multiple networks may co-exist in the same geographical area. Unfortunately, all these deployments will use the same ISM unlicensed band, and the networks will have to compete for the same radio ressource [14. so While channel hopping helps to combat external, narrow band noise, we may have, as we will highlight in this paper, complex interactions for different concurrent TSCH networks.

Typically, several Body Area Networks 15 and home automation [16 may use IEEE80215.4-TSCH in the same flat. Each network has its own border router to collect the traffic, and maintains its own schedule. Since the cells are allocated independently, the different networks may collide, impacting negatively the 35 reliability and the energy consumption. We propose here to tackle this kind of scenario.

In this paper, we propose to investigate the interactions between different, mutually interfering TSCH networks. We denote by TSCH instance a given network, comprising a set of devices attached to a controller (the border router), collecting the traffic. We will first study independent TSCH instances: they do not communicate with each other and allocate bandwidth independently. Then, we aim to explore the potential of a cooperative mechanism to reduce the amount of collisions and to make the performance more predictable.

Our main contributions are as follows:

1. we evaluate analytically and experimentally the impact of several TSCH co-located networks on the latency and reliability. We highlight the fact that synchronized TSCH instances help to reduce significantly the volume of collisions;

2. we propose a cooperative approach, where different border routers use their Enhanced Beacons to maintain a global synchronization. We keep on maintaining also the devices synchronized with their border router to avoid de-synchronizations;

3. we validate experimentally our synchronization mechanism, to handle a large number of colocated networks. A global synchronization is achieved step by step, even among interfering border routers which are not able to decode the Enhanced Beacons of each other.

\section{Related work}

We expect a wide adoption of the Industrial Internet of Things, leading to very dense deployments. While internal interference may be handled by carefully allocating transmission opportunities, external interference has also to be addressed. Slow channel hopping is a robust strategy to combat narrow band noise, and explains its adoption in the IEEE 802.15.4-TSCH mode[17.

\subsection{Coexistence of Multiple Networks}

Most of the IoT technologies use the same ISM unlicensed band. Thus, overlapping networks deployed for a different purpose have to compete for the same radio ressources. Some strategies have to be set up to reduce the amount of collisions, and to make the co-existence efficient. 


\subsubsection{Interference among Different Technologies}

Hayajneh et al. [18] provide a very good survey on the co-existence of different technologies for Wireless Body Area Networks (WBAN).

Several papers focus on the co-existence between Wi-Fi and IEEE 802.15.4 (Zigbee). Petrova et al. [19] study the impact of the packet size, the packet rate and the SINR on the packet losses for co-located IEEE 802.11 and IEEE 802.15.4 networks. If the channels of both networks overlap, the Frame Error Rate quickly increases (80\% in the worst case). Angrisani et al. 20 adopt a similar approach. They study also the impact of different packet inter-arrival times.

Liang et al. 21] try to localize experimentally the errors when Wi-Fi and Zigbee networks interfere. They remark that most colliding bits occur at the beginning of the frame. They propose to use error codes to make the transmissions more robust: the IEEE 802.15.4 packet is still decodable, even in presence of Wi-Fi interference. Park et al. 22 propose also a new mechanism to improve the performance in presence of Wi-Fi. Since the collisions of beacons strongly impact the global performance, the authors propose to use interference-free channels to transmit the beacons. The recent Bluetooth Low Energy (BLE) [23] is also negatively impacted by interfering Wi-Fi networks.

Hermans et al. 24 propose to classify the source of interference (micro-wave, Bluetooth, Wi-Fi) for an IEEE 802.15.4 network. By identifying the different sources of interference, the network should be able to adopt an accurate countermeasure strategy. For instance, a channel change may be sufficient when an interfering Wi-Fi Access Point is detected.

Ma et al. 25] investigate the interference arising with multiple Wi-Fi networks for smart utility networks. They only address internal interference by allocating orthogonal channels to each WLAN. Slow channel hopping aims rather to combat external interference, and requires different strategies.

The co-existence of Wi-Fi and IEEE 802.15.4-TSCH has already been studied in [26. Slow channel hopping is not sufficient to combat interference from the Wi-Fi networks: blacklisting has to be implemented to avoid repetitive packet losses. Local blacklisting is required to block a subset of physical channels for a given radio link [27].

In this paper, we focus rather on the co-existence of multiple networks using the IEEE 802.15.4-TSCH standard. We consider external interference generated by the same technology.

\subsubsection{Coexistence of Independent IEEE 802.15.4 Networks}

The beacon-enabled mode of IEEE 802.15.4-2006 proposes to use CSMA-CA, just after the reception of 90 a beacon to transmit the packets to the coordinator. However, collisions are very frequent, even with one single IEEE 802.15.4-2006 instance 28. Iraqi et al. also evaluate the number of collisions when different IEEE 802.15.4-2006 networks use different parameters (superframe duration, and beacon periods) [29.

To reduce the dirsuption, Sun et al. propose to listen beacons from neighboring BANs, and to modify the schedule to solve the collisions [30. DynMAC proposes to have a central controller which computes a global (31. The authors assume all the instances are owned by the same entity, and that interferences are a priori known. However, such approach may be inaccurate for independent deployments. All these solutions require also a global synchronization to avoid collisions.

INCA (Inter-Network Collision Avoidance) 32 focuses on the deployment of several IEEE 802.15.4 wireless personal area networks. When a PAN coordinator detects repetitive collisions, it shifts its wake-up time, so that both networks use non overlapping Contention Access Periods.

Torabi et al. 33 study analytically and experimentally the presence of multiple IEEE 802.15.4 networks. They propose to reserve space for alarm packets, so that a device may report repetitive erroneous beacons. The coordinator is in charge of changing accordingly the operated channel. Feeney et al. 34 simulate several co-existing Personal Area Networks (PAN) using the beacon-enabled mode of IEEE 802.15.4. They demonstrated the existence of long periods of disruption.

Alasti et al. [35] study multiple interfering Body Area Networks using a TDMA strategy. When colliding slots are detected, the coordinator is in charge of selecting randomly another slot for the corresponding child.

In this paper, we focus rather on the new TSCH mode of the last standard. Since it relies on TDMA 


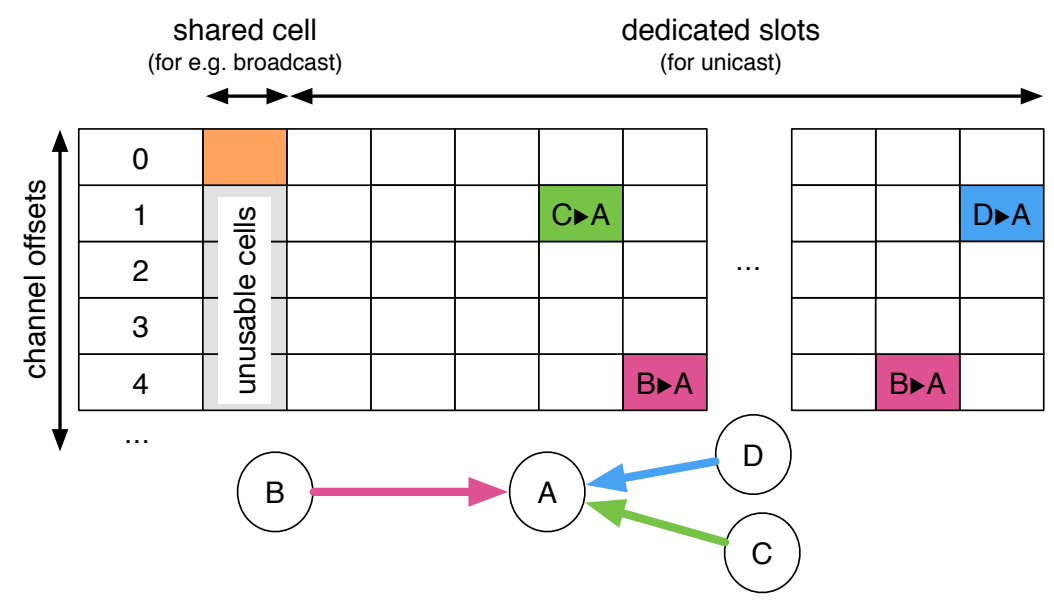

Figure 1: Schedule in a IEEE 802.15.4-TSCH network - illustration with 3 nodes and one border router

\subsection{IEEE 802.15.4-TSCH}

IEEE $802.15 .4 \mathrm{e}$ has published the TSCH mode for industrial low-power wireless networks 8 . To improve the reliability while maximizing energy savings, the network maintains a schedule, repeated periodically. TSCH uses a slotframe structure, constituted by a collection of timeslots, the slotframe length being configurable. The obtention of the schedule is out of the scope of the standard.

\subsubsection{Medium Access}

At the beginning of each slot, a node knows if it has to stay awake to receive or transmit a packet or if it may sleep to save its energy. A slot can be either dedicated (without contention) or shared (with a slotted Aloha mechanism to solve the conflicts between the contenders).

6 TiSCH minimal [36 advocates the usage of by default 1 shared cell, at the beginning of the slotframe, for an advertising purpose. Enhanced Beacons must be transmitted through the shared cells, so that all the synchronized nodes can receive them. With a dynamic schedule, additional cells are reserved on demand to forward the unicast data packets.

To improve the reliability, TSCH proposes to implement a slow channel hopping scheme. To each transmission opportunity is attached a channel offset. In the TSCH jargon, a cell is a pair of timeslot and channel offset. The channel offset is translated into a frequency to actually use at the beginning of each timeslot:

$$
\text { freq }=(A S N+\text { ch_offset }) \bmod 16
$$

where ASN is the absolute sequence number, ch_offset is the channel offset assigned to this cell in the node's schedule, with 16 physical channels, and mod is the modulo operator. If the number of channels and the slotframe length are mutually prime, we guarantee the same cell will use different frequencies in consecutive slotframes.

Let's consider 3 nodes (B, C, D) which transmit data to the border router (A) (cf. Fig. 1). Some shared cells (in orange) are dedicated for control packets on the channel offset 0 at the beginning of the slotframe. Because of the half duplex condition, all the links must be scheduled during different timeslots since they have a node in common (A). In our example, two cells are reserved for the transmissions from B to A.

While the common schedule avoids the collisions among the nodes inside a given TSCH network, colocated TSCH networks may collide with each other. We propose here to quantify this problem.

\subsubsection{Synchronization}

TSCH relies on a TDMA approach, and the nodes have to periodically re-synchronize to avoid large clock drifts and overlapping timeslots. Because the transmitter waits for a fixed offset after the beginning of the 


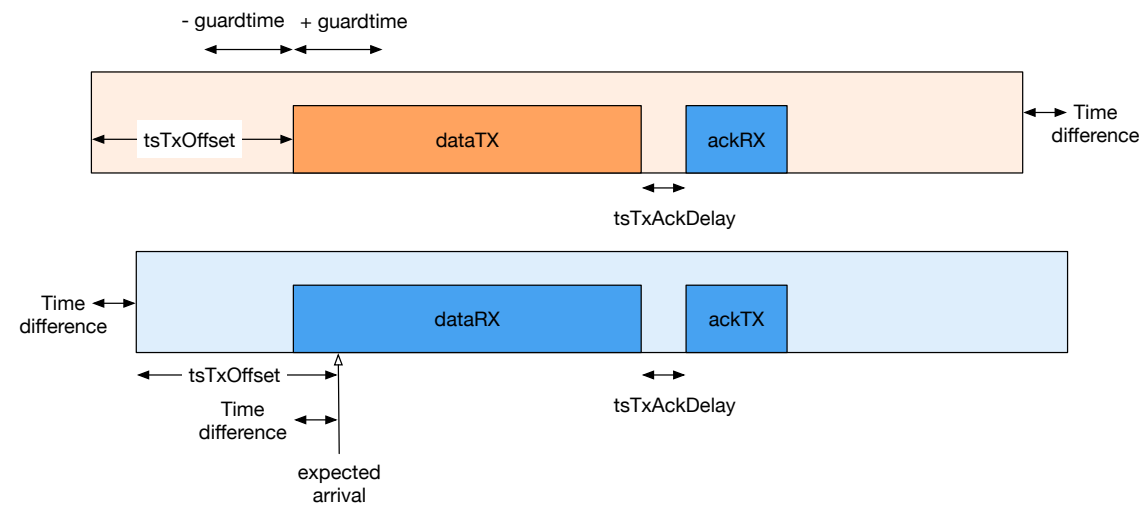

Figure 2: Synchronization mechanism for IEEE802.154-TSCH - computation of the time difference between the receiver and the transmitter

timeslot, the receiver is able to compute the time difference and to resynchronize itself. Typically, a node maintains a synchronization with its preferred parent when using RPL and 6TiSCH [37. If all the links (node $\rightarrow$ synchronizing parents) form a Directed Acyclic Graph, rooted at the border router, the network is globally synchronized.

Different synchronization mechanisms have been already proposed for distributed systems with real time clocks such as the global positioning system (GPS) and the IEEE 1588 precision time protocol (PTP) standardized in 38. However, these solutions have been designed for wired networks, and are inaccurate for low-power, unreliable networks.

Timing-sync Protocol for Sensor Networks (TPSN) 39] and the Time-Synchronized Mesh Protocol (TSMP) 40 have been proposed for this purpose. The robustness of TSMP leads to its adoption by ISA100.11a and Wireless HART protocols. It was designed to be deployed in a centralized network and it can achieve a very high accuracy, inferior to $1 \mathrm{~ms}$.

Because time differences are always present (i.e. the synchronization process cannot be ideal), TSCH provides a guard time before each transmission. The default tsTxOffset is set to $2120 \mu \mathrm{s}$ in the standard and the default guard time is $2000 \mu \mathrm{s}$ (Fig. 2). The actual time difference must be inferior to the guard time to maintain TSCH collision-free. This leads to sending at most a synchronization packet every 30 seconds when considering a typical value of $30 \mathrm{ppm}$ 41.

A node uses the Enhanced Beacons for the initial synchronization: it extracts the Absolute Sequence Number (ASN) - number of cells since the border router booted - to have an absolute time reference. Then, to resynchronize itself, a node uses two types of packets:

1. Data packets transmitted in unicast;

2. Beacons in broadcast.

Thus, explicit synchronization packets are required only when the data traffic is very low.

Stanislowski et al. 42 propose an adaptive synchronization. Indeed, the standard was designed to handle the worst case scenario: neighbors may actually resynchronize only if needed, reducing the number of control packets (beacons). Chang et al. 41 consider also the multihop networks. By appropriately selecting when to resynchronize, and by predicting the clock drifts with the parent, the authors propose to reduce the cumulative time difference, and this way to reduce the transmissions of beacons.

We focus here on synchronizing different TSCH networks. In particular, we don't have a hierarchy (i.e. a tree): a border router has to resynchronize itself with all its neighboring border routers. Besides, we consider high densities, and a pair of interfering border routers is separated by at most a few border routers. Similarly, we consider small and medium-scale networks, where the farthest device is typically 3 or 4 hops away of its border router. 


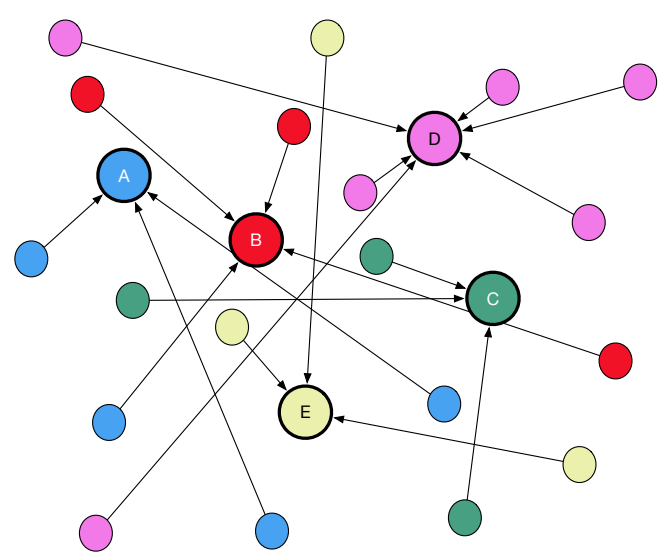

Figure 3: colocated networks - 5 different borders routers (a,b,c,d,e)

Let's first consider two TSCH networks perfectly synchronized with each other. They use for instance the same external synchronization process. This means that if the same cell is allocated twice, a collision

\section{Problem Statement and Theoretical Analysis}

IEEE 802.15.4-TSCH is designed to reduce the channel access contention and to deliver data with high reliability. However, the presence of colocated TSCH networks may suffer from interference. We concentrate in this study on the effect of sharing the same frequency band among coexisting TSCH networks.

For the sake of simplicity, we assume in this section, that the networks are deployed in the same location: any pair of transmitters (from any TSCH instance) collides for sure. We also assume that a collision occurs if the timeslots of the transmitters overlap, even partly. Thus, we assume all the data packets fill the timeslot, i.e. they have the maximum length.

We identified the two following scenarios:

Synchronized networks represent the most optimistic scenario. In other words, we have an ideal source of synchronization, and both TSCH instances use the same slotframe structure (i.e. length, number of shared cells);

noSync networks represent the most common scenario, where several instances are colocated but don't cooperate with each other. They have not the same ASN, and present clock drifts between the different instances. In particular, some cells may collide only in some of the slotframes (depending on their clock drifts).

We assume here that each network operates independently (Figure 3). Thus, the border router of each network assigns the cells to its devices. It applies a random strategy: it picks randomly a free cell (channel offset and timeslot) when a child asks for a new transmission opportunity. Obviously, the controller in the border router takes care of assigning different timeslots for the different children. Thus, collisions may only arise among nodes owning to different networks.

We present here a simple analytical model of the collision probability for several co-located, interfering TSCH network instances. In particular, in an ideal case, where all the TSCH slotframes are aligned, but each border router assigns independently the cells to its devices, how many TSCH networks could be deployed in the same area, without creating too many collisions, which both impact the reliability and the energy consumption? Similarly, we will expose the problems created by clock drifts when several networks are not synchronized with each other. We will study the relationship between the collision probability and the clock drifts.

This section aims to expose the problem of collisions created by several instances. We assume perfect radio links, and that all the nodes mutually interfere. 


\begin{tabular}{r|l} 
Variable & Signification \\
\hline $\mathcal{S}_{\text {coloc }}$ & set of co-located TSCH instances \\
$\mathcal{C}(i)$ & set of dedicated cells assigned to the instance $i$ \\
$N_{\text {choffset }}$ & number of channel offsets \\
$N_{\text {dedicated }}$ & number of dedicated timeslots \\
SLength & slotframe length (by default 101 in 6TiSCH) \\
$\Delta_{\text {clock }}(i)$ & actual clock drift (in seconds) per second \\
$\Delta_{\text {clock-max }}$ & maximum clock drift \\
$\Delta_{\text {tslot }}(i, j)$ & the drift in timeslots between two instances $i$ and $j$ \\
$T_{\text {tslot }}$ & Timeslot duration (by default 15ms)
\end{tabular}

Table 1: Formal notation used in the paper.

occurs in every slotframe. We aim here at analyzing the collision probability if both instances allocate their bandwidth independently.

\subsubsection{Analysis}

We consider a set $\mathcal{S}_{\text {coloc }}$ of colocated TSCH instances (cf. Table. 1 for the notation). The instance $i$ has a set $\mathcal{C}(i)$ of dedicated cells allocated for its unicast transmissions.

We focus on the collisions among dedicated cells. Let's consider a cell $c$ randomly chosen. Because the cells are assigned randomly, the collisions may occur during the dedicated part (shared cells have not to be considered). Thus, the probability that the instance $i$ chose it for any of its unicast transmissions is:

$$
\operatorname{Psel}_{\text {sync }}(i)=\frac{|\mathcal{C}(i)|}{N_{\text {dedicated }} * N_{\text {choffset }}}
$$

with $\mathcal{C}(i)$ the set of dedicated cells assigned for the network controlled by the border router $i, N_{\text {dedicated }}$ the number of dedicated timeslots in the slotframe, and $N_{\text {choffset }}$ the number of channel offsets. $P_{s e l}$ sync $(i)$ is related to the bandwidth pressure in the scheduling matrix. It designates how much bandwidth of the whole scheduling matrix is assigned to the network instance $i$.

Let's now compute the collision probability. We consider the TSCH instance $i$. The probability that one of its cells was also chosen by any interfering instance is consequently:

$$
\operatorname{Pcoll}_{\text {sync }}(i)=1-\prod_{k \in \mathcal{S}_{\text {coloc }}-\{i\}}\left(1-\operatorname{Psel}_{\text {sync }}(k)\right)
$$

\subsubsection{Numerical Results}

Figure 4 illustrates the impact of a dense deployments and the traffic intensity on the network performance. Many cells are available to schedule the transmissions (101 slots ${ }^{*} 16$ channels). Even in extremely harsh conditions (e.g. 10 networks which use a slotframe length of 101 and 30 dedicated cells), the ratio of collisions remains below $20 \%$. Thus, collisions and interference may arise mainly for very dense deployments and for a high traffic intensity.

This scenario assumes the different TSCH instances have the same time reference. A GPS may provide such accuracy but its energy consumption is very high, i.e. more than $150 \mathrm{~mW} 43$ which forbids its usage for battery powered border routers. Besides, a GPS only operates outdoor. Consequently, this synchronization scheme represents an ideal situation, unrealistic for most use cases.

\subsection{Asynchronous Instances (noSync)}

We now consider the case of several TSCH instances, which behave independently, i.e. not synchronized. Since the different instances derive in time, the collisions are time-variant. If initially two cells from two different instances are not sufficiently separated, the clock drifts may be sufficient to provoke a collision. 


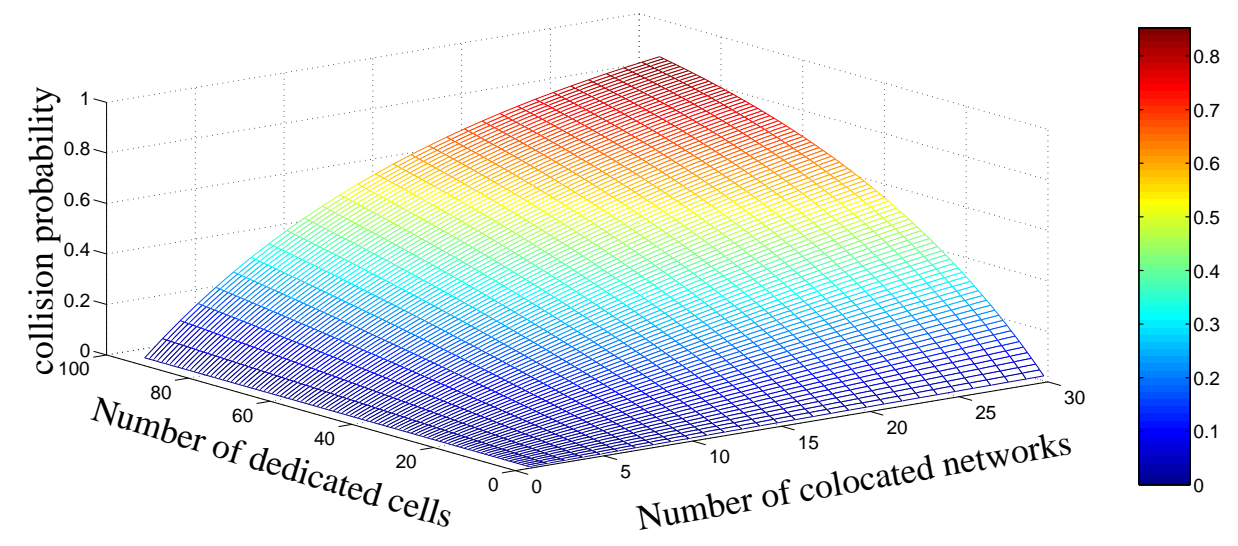

Figure 4: Collisions among different synchronized instances

For a sake of simplicity, we consider in this subsection that all the different instances use the same slotframe length (i.e. the same number of timeslots). The experiments we conducted in our testbed will highlight below in the paper that different lengths even exacerbate the problem of collisions.

\subsubsection{Analysis}

Let's denote by $\Delta_{c l o c k-m a x}$ the maximum clock drift per second. Two instances derive after the time $T$ by:

$$
T * \Delta_{\text {clock-max }}
$$

The crystal oscillator of MSP430 motes presents a typical accuracy of 30ppm [41]. Thus, $\Delta_{\text {clock-max }}$ is at most $30 \mu \mathrm{s}$. The actual time difference between two instances will be comprised after e.g. 10 minutes between $-36 m s$ and $+36 m s$ (i.e. approx. 2 timeslots by default). The probability of collision compared with the synchronous case (eq. 3) must consequently take into account this clock drift.

Without loss of generality, we fix now the reference clock as the clock of the first TSCH instance. The instance $i$ after the time $T$ has a timeslot difference compared to the reference clock:

$$
\Delta_{\text {tslot }}(i, T) \leq 1+\left\lfloor\text { Min }\left(N_{\text {dedicated }}, \frac{T * \Delta_{\text {clock-max }}}{T_{\text {tslot }}}\right)\right\rfloor
$$

$\Delta_{t s l o t}(k, t)$ denotes the (integer) number of timeslots by which the network instance $k$ has shifted from the time 0 to the time $t$, compared with the instance 0 . In figure 5 , the violet instance has a timeslot difference of 1 timeslot (17-16) at the time t. By definition, $\Delta_{t s l o t}(1, *)=0$

Let's consider a cell $c$ randomly chosen. The probability that this cell collides during the time $T$ for at least one transmission of the instance $i$ depends on the clock drifts. More precisely, it depends on the number of timeslots swept by this clock drift. Besides, since the slotframes are not required to be aligned, a collision may arise with any cell of the slotframe. Thus, the probability that at least one cell of the instance $i$ collides with the cell $c$ is:

$$
\operatorname{Psel}_{\text {async }}(i, T)=1-\left(1-\frac{\Delta_{\text {tslot }}(i, T)}{N_{\text {dedicated }} * N_{\text {choffset }}}\right)^{|\mathcal{C}(i)|}
$$

Finally, the collision probability that at least one transmission collides is:

$$
\operatorname{Pcoll}_{\text {async }}(i)=1-\prod_{k \in \mathcal{S}_{\text {coloc }}-\{i\}}\left(1-\operatorname{Psel}_{\text {async }}(k)\right)
$$




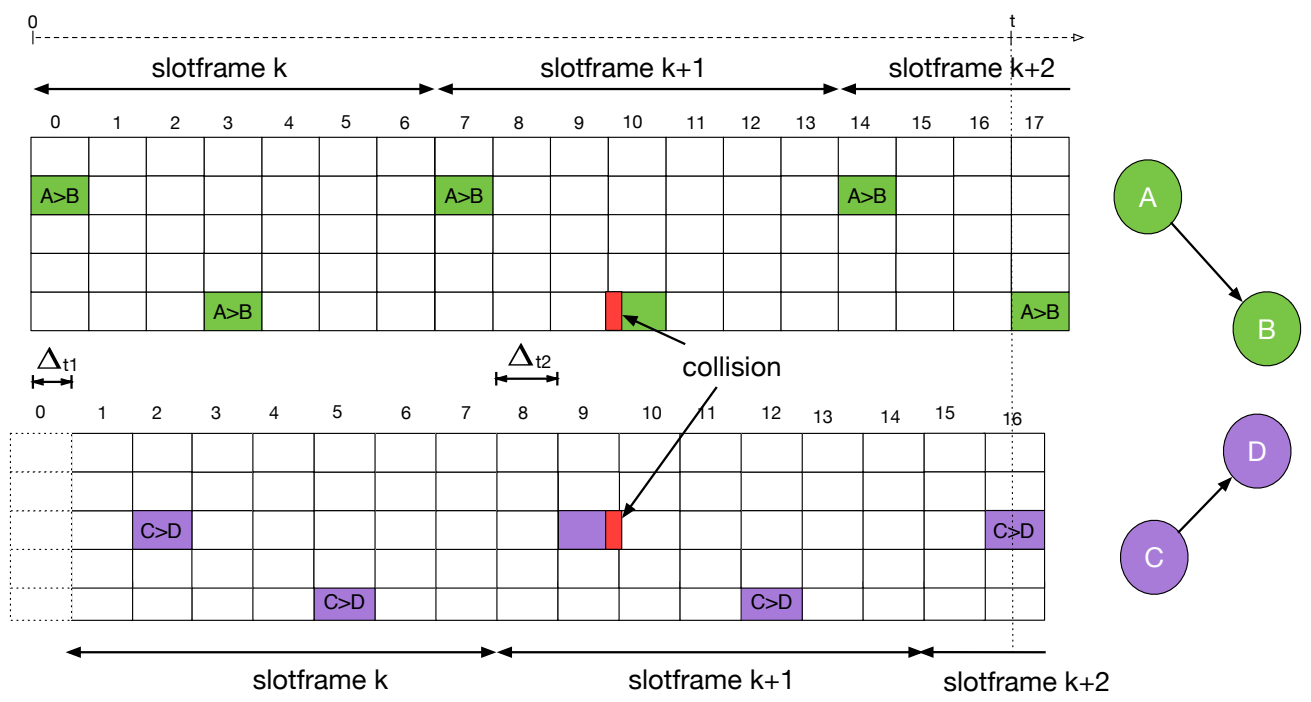

Figure 5: Two colocated networks with clock drifts

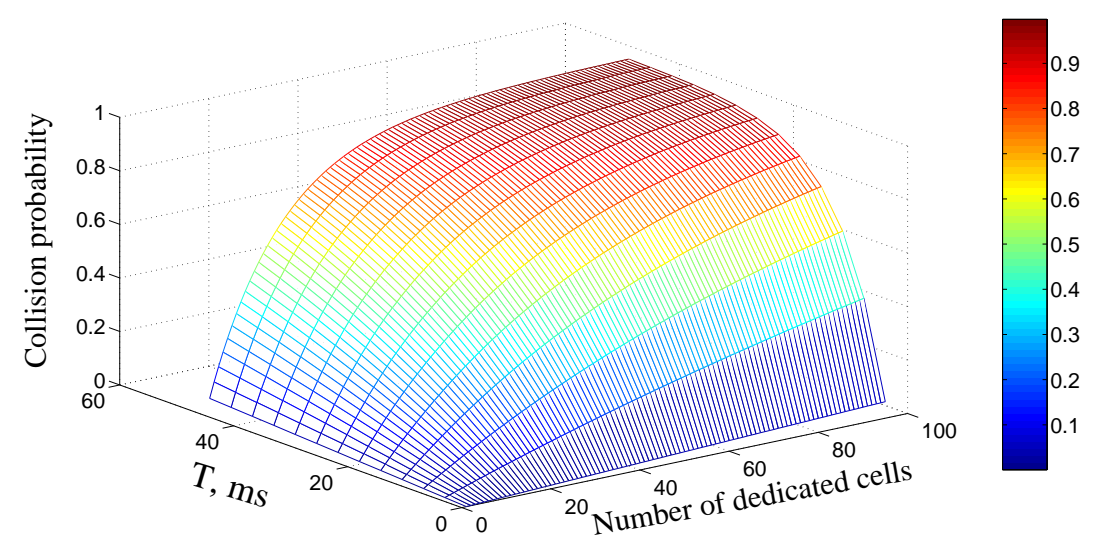

Figure 6: Collisions among different asynchronous instances, with a slotframe length of 101 slots, and 16 channels dock drifts. In the slotframe $\mathrm{k}+1$, two of the active cells overlap: since no
during the dedicated cells, the corresponding frames will collide for sure.

For the sake of illustration, we consider dozens of milliseconds for the clock drifts (Fig. 6). Typical clock drifts are at most $30 \mathrm{ppm}$ for crystal oscillators [41, and leads to a mutual drift of $3 \mathrm{~ms}$ after 50 seconds. Thus, a pair of cells will collide in several successive slotframes. These repetitive collisions have a very negative impact on the reliability, since several packets are dropped until the clock drift exceeds the timeslot length. In this paper, we propose to combat these black periods. 


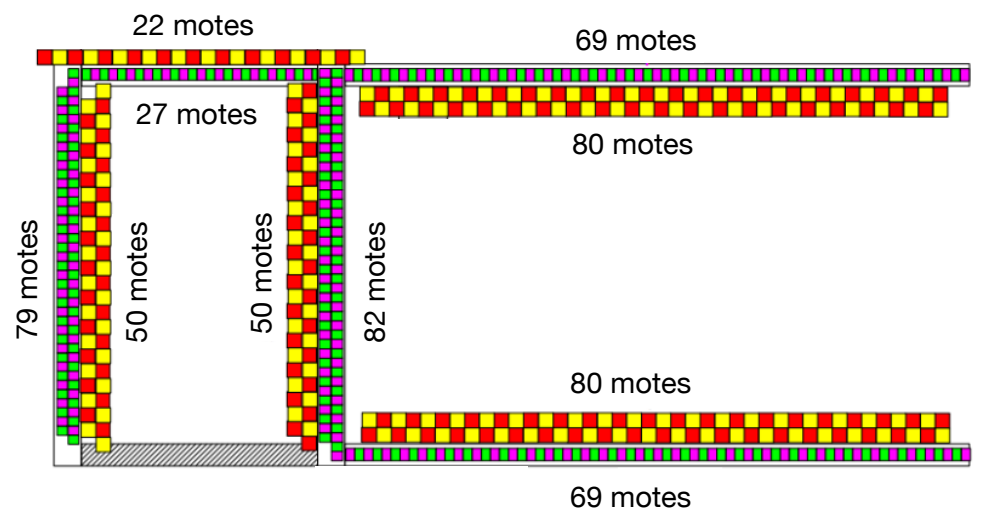

Figure 7: Grenoble FIT IoT-LAB testbed map.

\subsubsection{Blackout Periods, or Why Temporarily Overlapping Cells Matters?}

One may argue that clock drifts provide a positive effect when a pair of cells stops to collide when the

Number of scheduled cells: number of dedicated cells reserved by the Scheduling Function of $6 \mathrm{TiSCH}$ to transmit the data frames. More collisions mean also that the network has to reserve more transmission opportunities, leading to more idle listening and transmissions.

Number of retransmission: average number of link-level retransmissions. This represents a good first estimation of the collision probability of section 3 .

Blackout period: time during which a cell is colliding among two different TSCH instances. In other words, this correspond to the duration during which no packet is correctly received by the next hop for a given cell. 


\begin{tabular}{c|l|l} 
& Parameter & Default Value \\
\hline Experiment & Duration & 30 min \\
& Testbed organization & FIT IoT-Lab Grenoble \\
& Nb. of colocated networks & either four or variable \\
\hline CoAP & CBR (Unicast) & 1 pkts $/$ sec \\
& Payload size & 48 bytes \\
\hline RPL & DAO period & $50 s$ \\
& DIO period & $8.5 s$ \\
\hline \multirow{3}{*}{ TSCH } & Slotframe length & 101 \\
& NShared cells & 5 \\
& Timeslot duration & 15 ms \\
& Maximum retries & 3 \\
& Schedule & Random Assignement \\
\hline Queues & Timeouts & $8 s$ \\
& Queue size & 10 packets \\
& incl. data packets & at most 6 packets \\
\hline Hardware & Antenna & Omnidirectional \\
& Radio chipset & AT86R231 \\
& Node & ST2M32F103REY
\end{tabular}

Table 2: Experimental setup.

\subsection{Experimental Setup}

We used the FIT IoT-LAB platform ${ }^{1}$, which is part of FIT ${ }^{2}$, an open large-scale and multiuser testing infrastructure for IoT-related systems and applications. Our study was conducted over the testbed located in Grenoble's site (cf. Fig. 7). This testbed belongs to the real-world testbed category, since several WiFi Access Points (APs) are deployed in the building. Under such a realistic indoor environment, i.e. a typical office space, the nodes are subjected to external interference originated from wireless devices using other technologies, such as Wi-Fi (2.4 GHz band).

As depicted in Fig. 7. this testbed consist of 380 nodes deployed in an area of $65 \mathrm{~m} \times 30 \mathrm{~m}$. Most of the deployed sensor nodes (i.e. 90\%) are placed under the raised floor, while the remaining $10 \%$ are deployed above the dropped ceiling. In our experimental campaign, we employed the M3 nodes, based on an STMicroelectronics 32-bit ARM Cortex-M3 micro-controller (ST2M32F103REY). It embeds an AT86RF231 radio chip, providing an IEEE 802.15.4 compliant PHY layer. In this set of experiments, we select nodes which all hear each other.

To conduct our experiments, we employed OpenWSN 3 an open-source implementation of a full protocol stack based on loT standards (IPv6, 6TiSCH, 6LoWPAN, UDP, RPL, CoAP). In particular, we used the modified implementation of OpenWSN 4 to provide distributed scheduling [44. A node chooses to synchronize with its RPL parent, as $6 \mathrm{TiSCH}$ advocates.

Default parameters values are depicted in table 2

\subsection{Homogeneous vs. Heterogeneous Scenario}

IEEE 802.15.4-TSCH has to support a large variety of traffic loads to prove its efficiency in most of the situations. For colocated networks, we make the distinction between the following two scenarios:

\footnotetext{
${ }^{1}$ https://www.iot-lab.info/

2 https://fit-equipex.fr/

3 https://openwsn.atlassian.net/

${ }^{4}$ https://github.com/ftheoleyre/openwsn-fw/

5 https://github.com/ftheoleyre/openwsn-sw/
} 


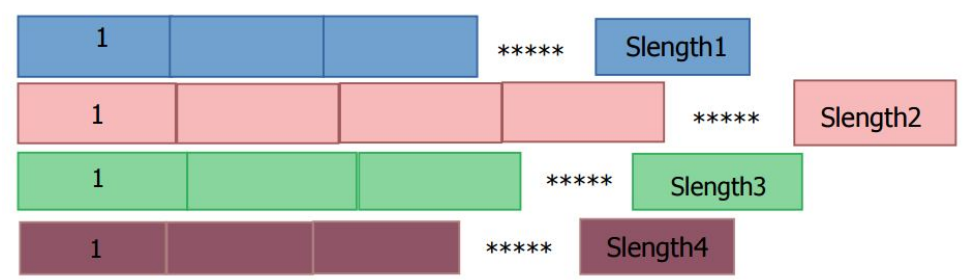

Figure 8: Illustration of different slotframes length configuration.

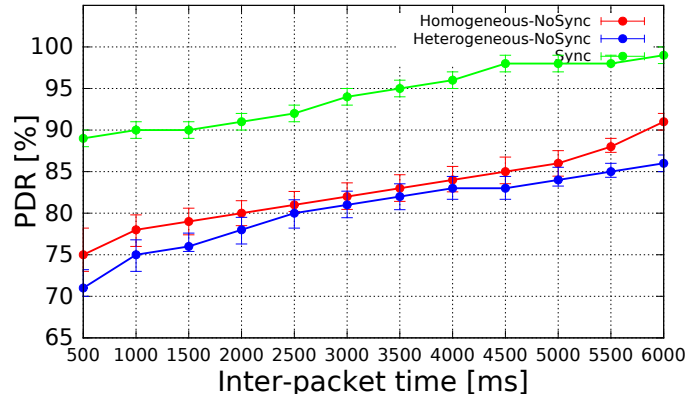

(a) Link-LayerPacket Delivery Rate

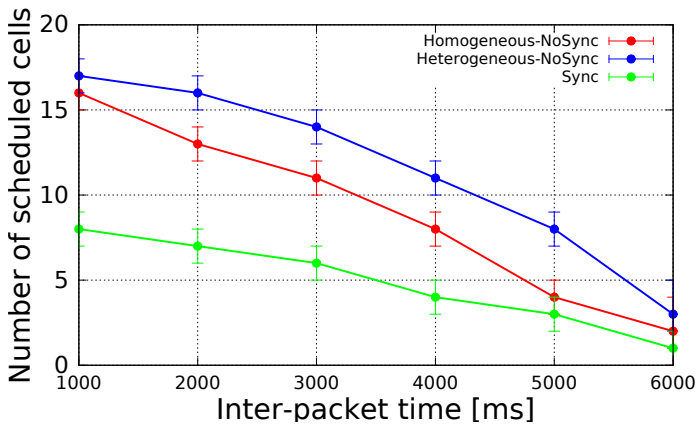

(c) Number of scheduled cells (energy efficiency)

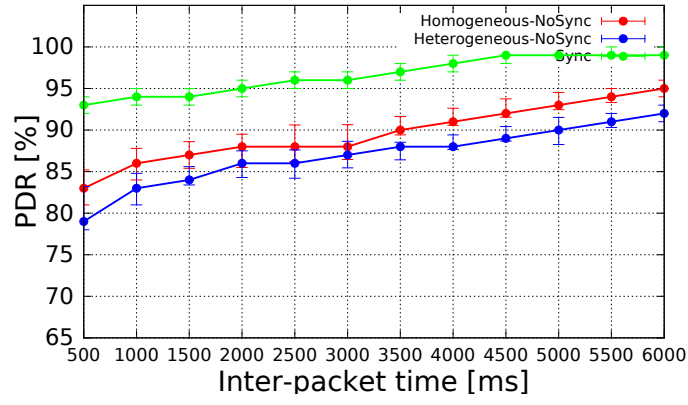

(b) Network-Layer Packet Delivery Rate

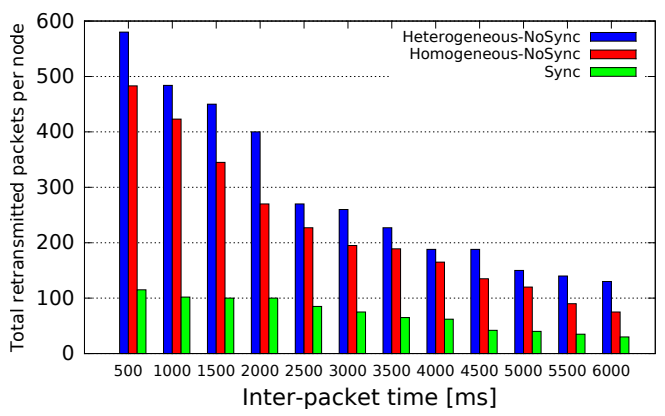

(d) Total number of retransmissions

Figure 9: Performance of Synchronized network vs. unsynchronized networks- 4 TSCH instances and 2 nodes per border router

homogeneous: all the different TSCH networks are configured similarly, using in particular the same slotframe length;

heterogeneous: different TSCH instances may use different slotframe lengths, because e.g. the periodicity of their traffic is different. Let's consider networks with 4 different slotframe lengths (cf. Figure 8). Since all the networks operate independently, the slot boundaries are not aligned. Moreover, the cycle of the repetition is not identical for all the slotframes, which means that a cell for a small slotframe length may collide only during some of the slotframes.

To compare these scenarios, we measured the performance with an inter-packet arrival comprised between $500 \mathrm{~ms}$ and 6,000 ms. The Scheduling Function allocates independently the cells, selecting randomly one channel offset and timeslot when a cell has to be allocated. We re-used the local scheduling function presented in [44. We run experiments for 30 minutes and we plot 95 confidence intervals.

Figure 9 compares the results obtained with respectively perfectly synchronized networks, homogeneous without synchronization (same slotframe length) and heterogeneous without synchronization (different slotframe lengths).

In the nosync case, each border router behaves independently and may start its slotframe at any time. 
The different border routers boot automatically after flashing their firmware. Since they are flashed sequentially, their slotframe are not synchronized (i.e. they have a different ASN value, and their timeslots are not aligned).

We first measure the Link-Layer Packet Delivery Ratio. A smaller inter-packet time means more packets to forward, and possibly more collisions (Fig. 9a). The synchronized scenario achieves a very high PDR for small loads (one packet every 4 seconds). And it keeps on providing a 90\% packet delivery ratio with 1 packet per second. When the networks are not synchronized, the impact on the PDR is more important. And finally, heterogeneous configurations tend to also increase the impact of collisions (because of the different periods, collisions are more anarchical). With only one packet every $6 \mathrm{~s}$, the heterogeneous case achieves a PDR of $90 \%$ (vs. $100 \%$ in the homogeneous scenario).

Moreover, we report in (Fig. 9b) the network-Layer Packet Delivery Ratio, i.e. the ratio of packets delivered to the next hop after at most 3 retransmissions by the MAC layer (the default value defined in IEEE 802.15.4-TSCH). $100 \%$ of the packets are delivered in the synchronous case with an inter packet time superior to $4.5 \mathrm{~s}$. With high traffic, almost $95 \%$ of the packets are correctly delivered after at most 3 link-layer retransmissions. On the contrary, the noSync cases don't succeed to deliver all the packets, even with a very low traffic: collisions are present in successive slotframes, and the transmission fails in every cell.

We also measured the number of cells scheduled by the local scheduling algorithm (Fig. 9c). The Scheduling Function allocates more cells when it has too many packets to transmit compared with the number of allocated cells. In particular, a low PDR for a given cell leads the Scheduling Function to reserve new cells in the schedule. Thus, collisions have an impact on the PDR and the number of allocated cells.

We finally measured the number of link-layer retransmissions (Fig. 9d): more retransmissions mean also a larger energy consumption. Without surprise, the synchronous case is the most efficient: less collisions mean more reliable cells, with less retransmissions. The number of collisions is larger for the noSync case: more cells collide, and require to retransmit the corresponding packets.

In conclusion, having heterogeneous configurations and without synchronization does finally impact both the reliability and the energy efficiency. In the rest of the paper, we assume that all the TSCH instances use the same slotframe length. This way, the collisions are repetitive, and easier to detect and to solve.

\subsection{Blackout Period}

To better estimate the amount of collisions, we evaluate experimentally the impact of the Inter-packet time on the blackout duration. We simulate here a scenario where two TSCH instances are co-located and their slotframes are statically configured: the shared cell os used for broadcast packets, and a fixed dedicated cells os used to transmit the unicast data packets. We deactivate the transmission of DIO and DAO to not disturb the measures the reliability for data packets.

Fig. 10 illustrates the duration of the blackout periods, i.e. time interval during which no data packet is delivered to the next hop. This duration may last between 100 and 150s (for the largest traffic rates). During this blackout period, no packet at all can be delivered, which is very prejudicial for time sensitive traffic.

\section{Synchronizing Different IEEE 802.15.4-TSCH Instances}

As we highlighted, different colocated networks are much more efficient when they are synchronized with each other. This way, we make the collisions repeatable, and predictive. A Scheduling Function may be able to detect the colliding cells, and to re-allocate them until no collision arises.

\subsection{Clock Accuracy and Slot-based Synchronization}

In the bootstrap phase, the nodes extract the Absolute Slot Number (ASN) from beacon. It also computes the timeslot boundaries from the time of arrival of the beacon, neglecting the propagation delay of the radio signal. Thus, the node is able to adjust its clock, with the absolute clock value of the source. 


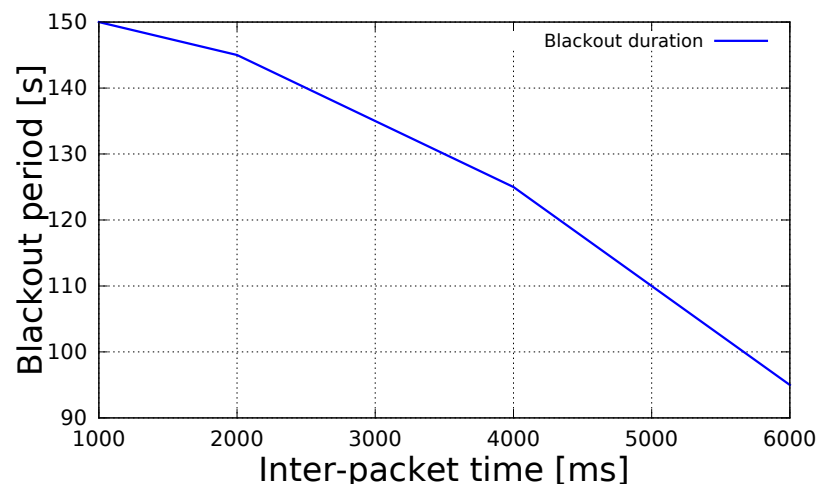

Figure 10: Impact of the Inter-packet time on the blackout duration-two TSCH instances and one node per border router

The accuracy of the crystal based oscillator may be affected by physical factors like temperature, humidity or by differences in manufacturing [45. Thus, the re-synchronization has to be sufficiently frequent to maintain the clock drifts under the maximum value. In particular, the re-synchronization period depends on two main factors as depicted in (Eq. 8) :

$$
\text { Period }_{\text {resync }}=\frac{\text { Guard }_{\text {time }}}{2 * \operatorname{Clock}_{\text {drift }}(\text { max })}
$$

with Guard $_{\text {time }}$ being the guard time of the standard $\left(2000 \mu \mathrm{s}\right.$ in TSCH), and Clock $_{\text {drift }}($ max $)$ the maximum clock drift as defined by the manufacturer (typically $30 \mathrm{ppm}$ ). With these default values, a node has to resynchronize at most every 30 seconds.

IEEE 802.15.4-TSCH defines two slot based synchronization mechanisms [46] to reduce the difference of time between a transmitter and a receiver:

Frame-based Synchronization: the receiver aligns its slot edges according to its source of time based on the TsTxOffset and the instant of data reception. More precisely, the clock of receiver is adjusted to match that of its neighbor as explained in (Fig. 2);

ACK-based Synchronization: the source of time saves the instant of data reception. This value is added in the acknowledgment sent back. The sender uses this information to shift its clock and to synchronize with the receiver.

We will here re-use this mechanism, and adapt it to deal with several independent border routers.

\subsection{Requirements and Considerations}

We have shown that collisions become predominant when many unsynchronized networks share the same frequency band. To face these limitations, several challenges have to be considered when proposing new synchronization algorithms to manage dense deployments:

Energy consumption: since we consider an unsynchronized scheme, IEEE 802.15.4-TSCH networks do not share the same ASN, and the slot boundaries are not aligned. The reservation of new cells should be minimized because it consumes energy. Similarly, we have to reduce the amount of collisions by realigning the slotframes of different border routers;

High densities: we have to support large densities, where many instances compete for the same radio resource;

No specific hardware: deploying expensive (financially and energetically) hardware like GPS receivers is not an efficient solution for synchronization; 


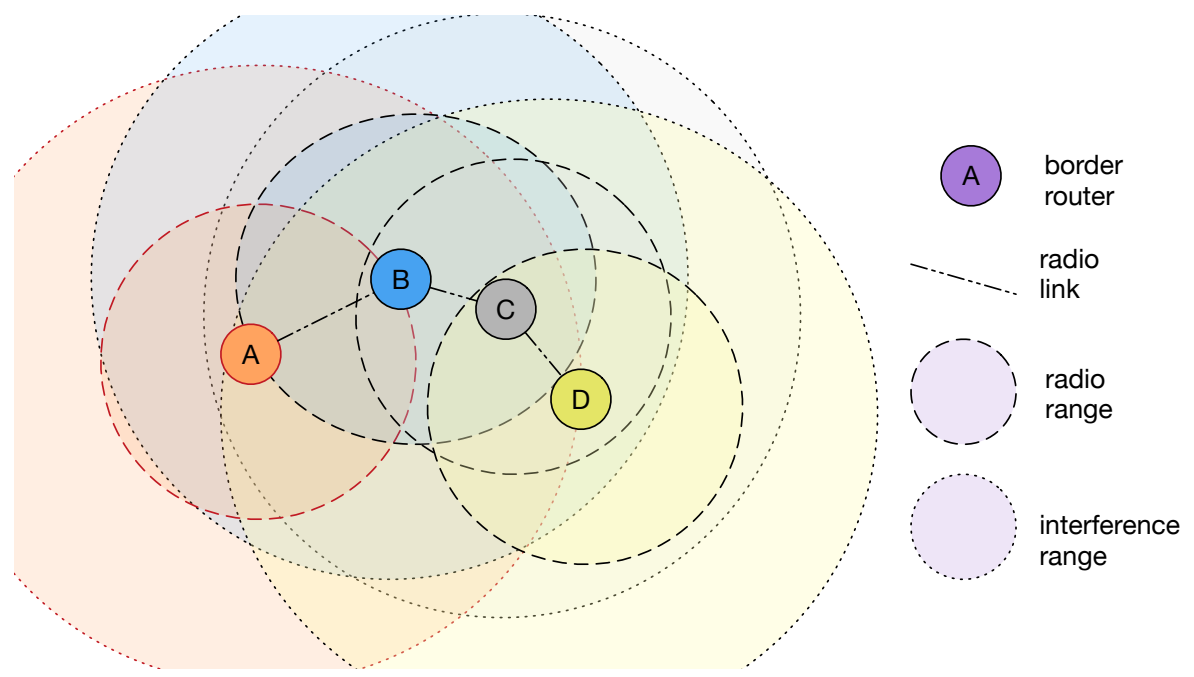

Figure 11: Transitive connectivity of a pair of interfering border routers

Convergence Speed: since we consider a large-scale scenario, we should reduce the time spent to ensure synchronization across the different networks;

Traffic Adaptative: the schedule is adjusted locally by the border router as soon as a device has more traffic to forward. A global controller for all the network seems not recommended.

To achieve these goals, we provide here a cooperative strategy, so that the slotframes of the different networks are realigned through a distributed synchronization. So, we can propose easily a new mechanism to identify colliding cells, and to modify locally (uncooperatively) the schedule to solve these collisions.

\subsection{Assumptions}

We consider here high densities: several TSCH networks are deployed in the same location, and mutually interfere. To maintain a global synchronization, the different borders must hear each other, and decode their Enhanced Beacons. More precisely, we assume that any pair of interfering border routers is connected through a chain of border routers, able to pairwise decode their Enhanced Beacons. A pairwise synchronization of the different border routers can lead to a global synchronization [47.

Let consider the figure 11. The border routers A and B mutually interfere. We assume that the density is sufficiently high so that the chain of border routers $\mathrm{A} / \mathrm{B} / \mathrm{C} / \mathrm{D}$ exists: with a local synchronization, the network is finally globally synchronized. The border routers have to send periodically Beacons to maintain a pairwise synchronization. Since the time difference is cumulative along a chain of border router, the beacon's period has to be linear with the maximum hop distance between two interfering border routers. Practically, we highlight in the performance evaluation that this time difference is limited, and we can maintain good performance, even for long chains of border routers.

Besides, we typically target small-scale (e.g. Body Area networks [15]) and medium-scale (home automation [16]) topologies. The nodes inside the same TSCH instance may use the data packets to resynchronize themselves, and the time difference between the border router and its farthest nodes is largely inferior to the guard time.

\subsection{Synchronization of Different TSCH Instances}

We provide here a mechanism to re-synchronize explicitly several instances to maintain the performance stable. To reduce the energy consumption, we simply re-use the Enhanced Beacons. If the EB have 


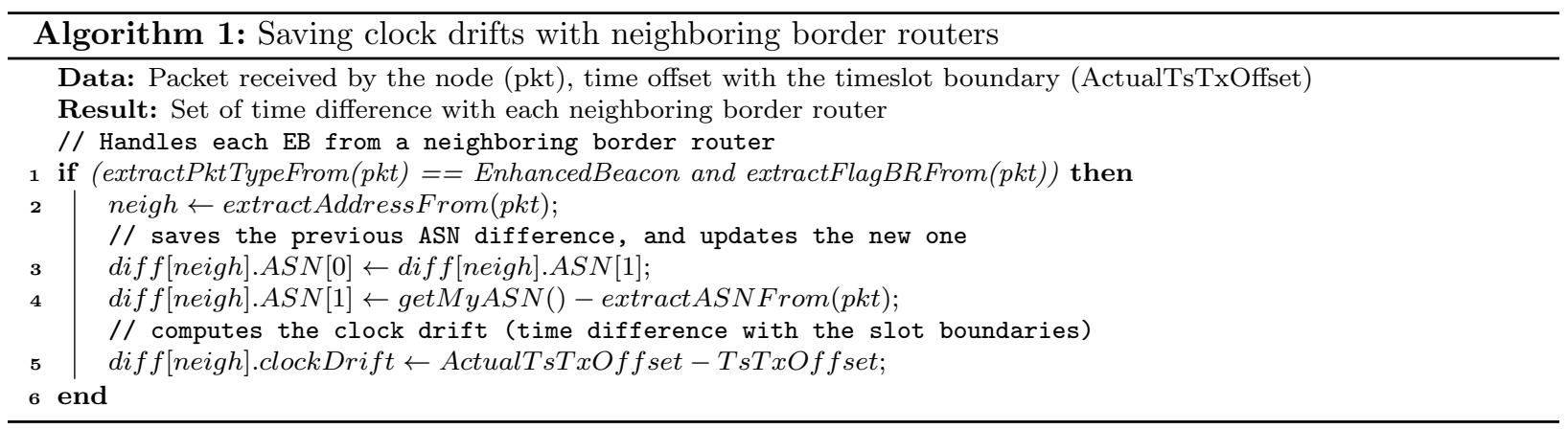

to be ciphered, dedicated non-ciphered EBs have to be used to synchronize different networks. This resynchronization mechanism is able to limit clock drifts and to maintain the ASN difference constant among different border routers.

Let us consider $\mathrm{N}$ co-located networks with $\mathrm{N}$ distinct border routers. The border router is the root of its synchronization tree, and computes the schedule for its network. All the nodes which are attached to this border router form an instance, sharing the same clock. Each border router has to execute the following steps:

1. track all the Enhanced Beacons from its neighboring border routers (and compute the clock drifts);

2. compute its ideal own clock based on the time difference with neighboring border routers;

3. upper-bound the clock re-adjustment to maintain its own network connected.

\subsubsection{Step 1: Computing clock drifts with neighboring border routers}

Only the border routers adjust the clock of their network, thus they have to maintain a list of their neighboring border routers. They record the time of reception of each EnhancedBeacon, and the corresponding

ASN. Each EnhancedBeacon contains the ID of the network, its ASN value. Thus, the receiver proceeds as follows (Algo 1):

1. it extracts the ASN and the ID of the source; If no entry corresponds in its neighborhood table, it inserts a new one;

2. it computes the time difference from the beginning of the timeslot, exactly like the normal TSCH procedure for non border routers (Fig. 2), and stores the value in the table;

3. it computes the ASN difference between itself and the source, and stores it as the new ASN difference (saving the previous one).

\subsubsection{Step 2: Resynchronization with the neighboring border routers}

A node has to compute the average difference with all the neighboring routers (itself comprised). It leads to an ideal centroid to which the node's clock has to converge. This synchronization scheme has been proved to converge quite well in distributed systems [48, [49]. To compensate the clock drifts, we have to consider:

Slot boundaries alignement: obviously, the cells of different networks should not overlap, to minimize the number of collisions, as exposed in the noSync scenario (cf. Fig. 9):

ASN difference: the ASN difference should be maintained constant among the different border routers. If the slotframe size is the same for all the nodes, this means that the collisions are repetitive, easier to detect and alleviate;

Connectivity: the border router should keep being synchronized with its network. In particular, the actual clock drifts with its children should not exceed the guard time, else, the devices will become disconnected, engaging a long and expensive procedure to re-discover the border router. 


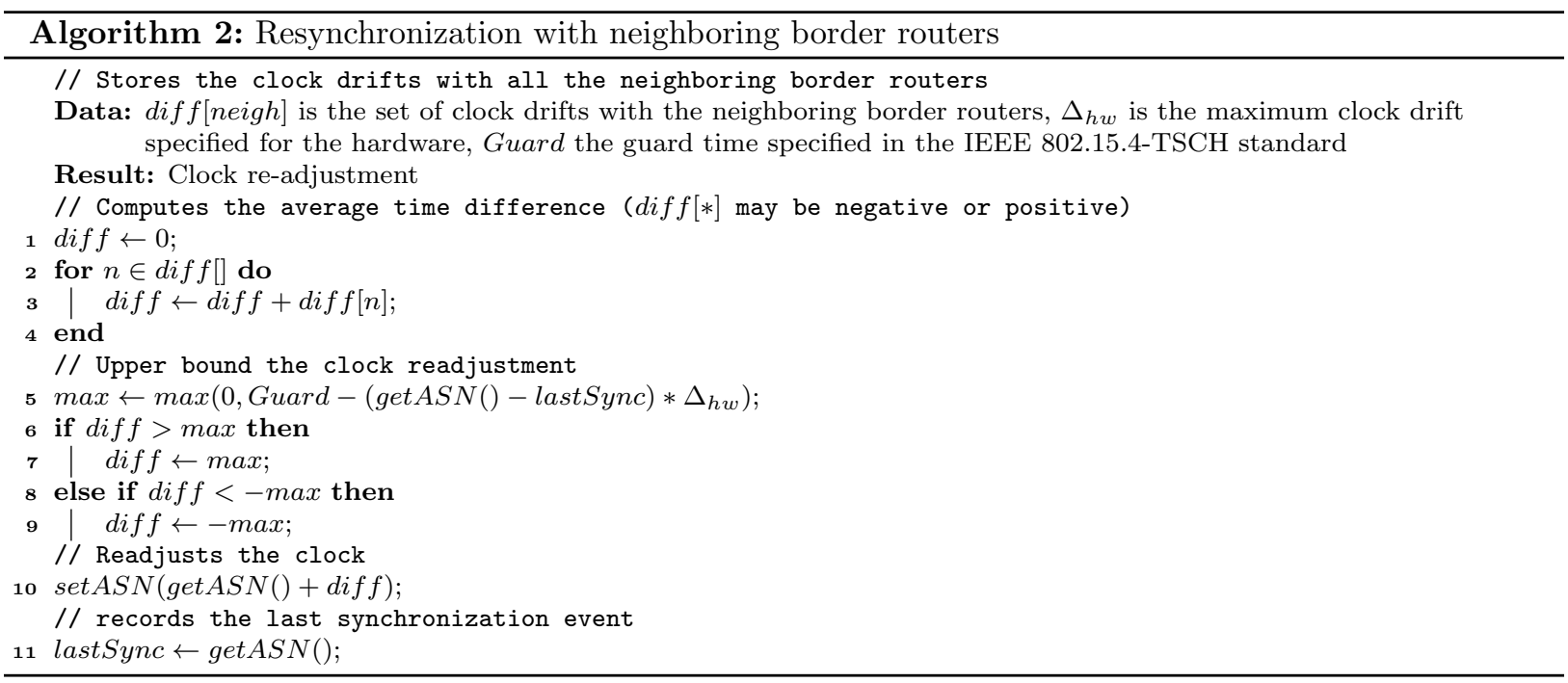

A frequent transmission of Enhanced Beacons would accelerate the convergence of the synchronization mechanism: the devices can re-adjust more frequently their clock, while maintaining the time difference below the guard time.

Algorithm 2 defines formally our approach. Until the border router is ready to send the next beacon, it overhears beacons sent by neighboring coordinators. Just before transmitting its own EB, it computes the centroid of the clocks of its neighbors (itself included) (lines 2-4) It also upper bounds the clock readjustment based on the time at which it transmitted the last EB, and the guard time (line 5).

This synchronization scheme operates well with any number of neighbors and any topology [48. Each time an EB is transmitted, the concerned border router readjusts its clock, becoming closer to the centroid. Step by step, all the independent instances become aligned, with a constant pairwise ASN difference.

\section{Performance Evaluation}

We conducted a thorough experimental campaign to validate the behavior of our proposition. The different networks must be able to cooperate to reduce the number of collisions, and even to remove the blackout periods during which the cells overlap in successive slotframes, and lead to repetitive packet losses.

We re-used here the experimental setup as described in section 4.1. We consider a distributed network composed of a variable number of instances which have independent border routers. In particular, they don't rely on an external source of synchronization such as a GPS.

We compare the two following solutions:

NoSync: all the TSCH networks behave independently, allocate autonomously their cells, and detect colliding cells to reallocate them;

SynCoop: each border router allocates independently the bandwidth in its instance. However, it also executes the algorithm 2 to quantify the clock difference with the neighboring border router, and to re-align globally the slotframes.

\subsection{Efficiency and Scalability}

We first study the reliability achieved with multiple colocated networks, when the cooperative synchronization mechanism is activated or not (Figure 12). To quantify the reliability, we measure the Link-Level Packet Delivery Ratio. With 2 colocated networks, SynCoop maintains a very high reliability (99\%) by 


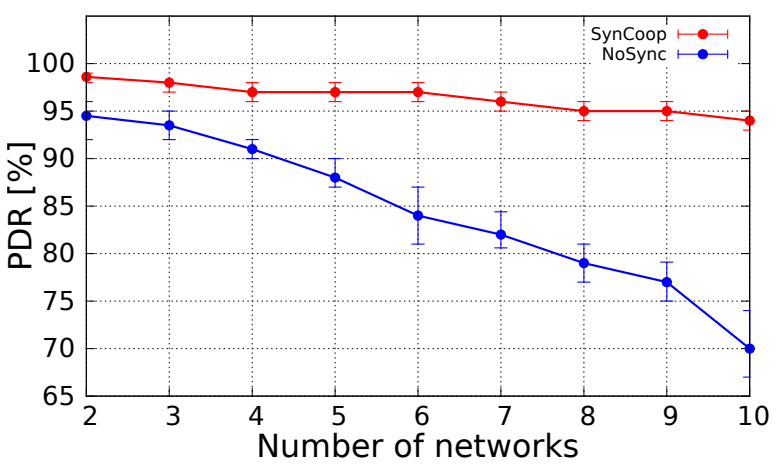

(a) Impact of the number of colocated networks, inter packet time of $6,000 \mathrm{~ms}$

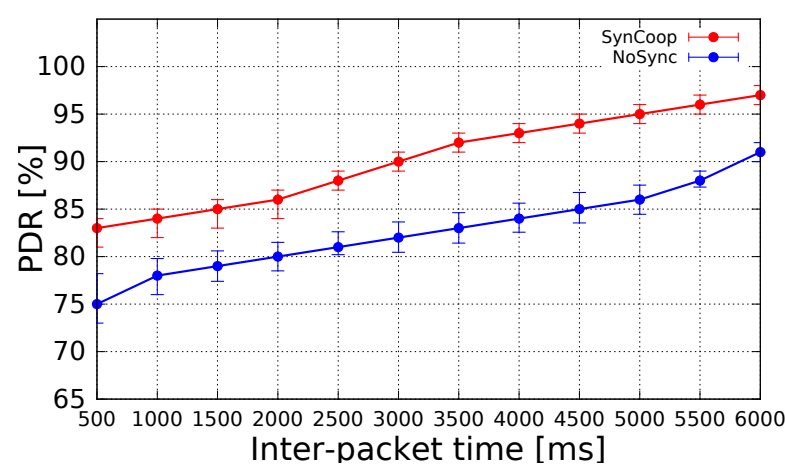

(b) Impact of the traffic intensity, 2 colocated networks.

Figure 12: Average Link-Layer Packet Delivery Ratio evaluation (proposed scheme vs. unsynchronized networks scenario).

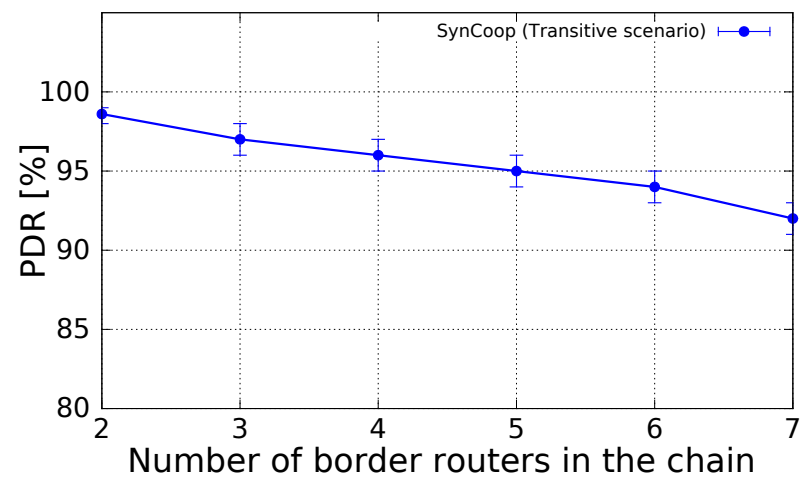

Figure 13: Impact of the chain's length between two border routers on the Packet Delivery Ratio

re-aligning the slots. The Scheduling Function is efficient to then detect colliding cells, and to reallocate them elsewhere in the slotframe.

Even in very harsh conditions (more than 10 colocated networks, which mutually interfere), we maintain a very high PDR: more than $95 \%$ of the packets are delivered correctly to their border router. Without synchronization, collisions become predominant, and only $70 \%$ of the packets are delivered.

\subsection{Transitive Synchronization}

To evaluate the robustness of our synchronization mechanism, we also evaluate the impact of the length of the chain between a pair of interfering border routers (Fig. 13). Since the time difference is cumulative, the two interfering border routers must be sufficiently tightly synchronized to keep on avoiding collisions.

We now measure the link-level packet delivery ratio when we increase the number of routers in this chain. When the border routers are able to decode their EB, $98 \%$ of the packets are delivered. When this pair needs 1 intermediary router to resynchronize itself, $97 \%$ of the packets are delivered. Under the harshest conditions ( 5 border routers separate the pair), 93\% of the packets are delivered, as compared with the $82 \%$ delivery ratio in Fig. 6.3 in the NoSync scenario. SynCoop realigns the frames even when the different TSCH instances need a transitive synchronization.

\subsection{Convergence}

To evaluate the convergence of our solution, we measure the clock re-adjustments of a border router which tries to synchronize with 3 other neighboring border router (while keeping on transmitting data packets with its children). Figure 14 represents the cumulative number of ticks, averaged during a given period (1,308 


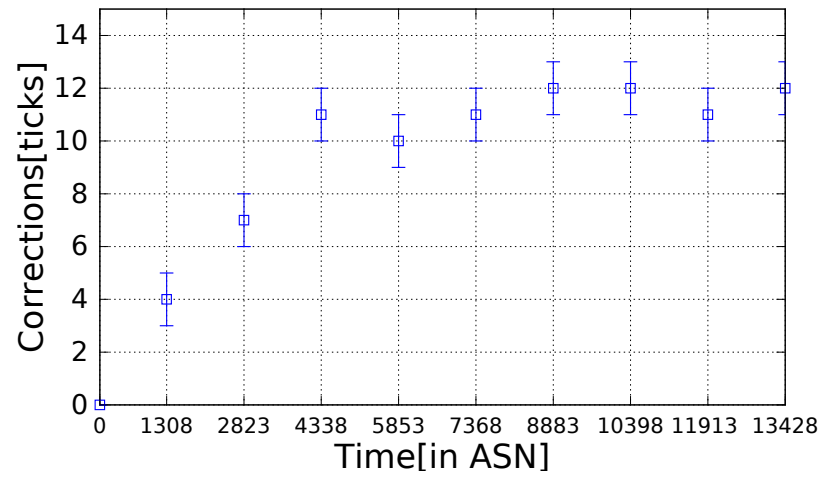

Figure 14: Convergence - Clock adjustments with 4 colocated networks

slots $=19$ seconds). The border router needs to first discover the other border routers. Since slow channel hopping is used, the two border routers may take time to receive their Enhanced Beacons. The first border router is discovered after 1,308 slots $(=20 \mathrm{~s})$, and the node keeps on synchronizing with the two other border routers, discovered later. After 4,000 slots (=1min), all the border routers have been discovered, and keep on pairwise resynchronizing.

We can remark that only a small cumulative number of ticks is needed to resynchronize the different instances. So, a border router doesn't lose the synchronization with its children. Since only the slot boundaries have to be aligned (and the absolute time difference does not matter), and because the guard time is sufficient, on average 12 ticks are sufficient. Besides, we noted that no device becomes desynchronized during the experiments. All of them send a sufficient number of data packets and/or receive enough Beacons to resynchronize themselves.

\subsection{Efficiency}

To have an estimation of the energy efficiency, we measure in Figure 15 the number of cells during which nodes are transmitting or receiving. A node has to wake-up in all its active cells to possibly receive a frame, consuming mechanically more energy. .

We first evaluate the impact of the traffic intensity (Fig. 15a). A smaller inter packet time means a larger number of packets to forward, and a larger number of cells to schedule. However, SynCoop achieves both a lower number of cells and a larger reliability (Fig. 6.3). Intuitively, less reliable cells means that the Scheduling Function has to increase the number of cells to reserve while not achieving to alleviate entirely the negative impact of the retransmissions on the reliability.

When considering the impact of the number of colocated networks (Fig. 15b), we lead to the same conclusions. SynCoop needs less cells while providing a higher reliability. Even with 2 colocated networks, we need twice as much bandwidth without synchronization (1 vs. 2 cells).

Finally, we measured the average number of retransmissions required for both solutions (Fig. $15 \mathrm{c}$ ). Indeed, a larger number of retransmissions means a larger number of cells to allocate and/or more transmissions which consume also energy (even if the number of cells remains unchanged). Without synchronization, the collisions are more frequent, increasing mechanically the number of transmissions required to deliver the packets. However, synchronizing the multiple instances helps to reduce this collision probability, particularly, for heavy loaded conditions.

\subsection{End to End Delay}

We finally measured the end-to-end delay (Fig. 16). More collisions mean also more retransmissions. Since the scheduler assigns randomly the cells to use, a retransmitted packet has to be buffered until the next transmitting cell. Thus, NoSync presents a larger end-to-end delay. On contrary, SynCoop achieves a lower delay, by using non colliding cells. The re-allocation mechanism of the scheduling function is efficient to detect collisions and re-allocate the corresponding cells. 


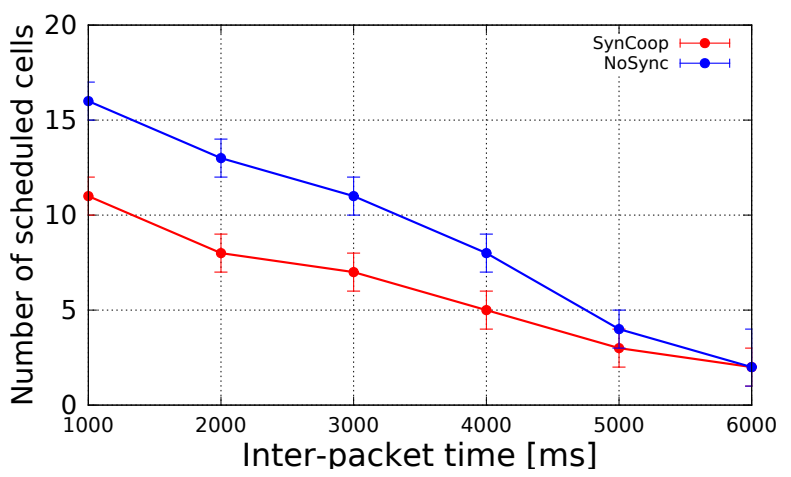

(a) Number of scheduled cells vs. Interpacket time

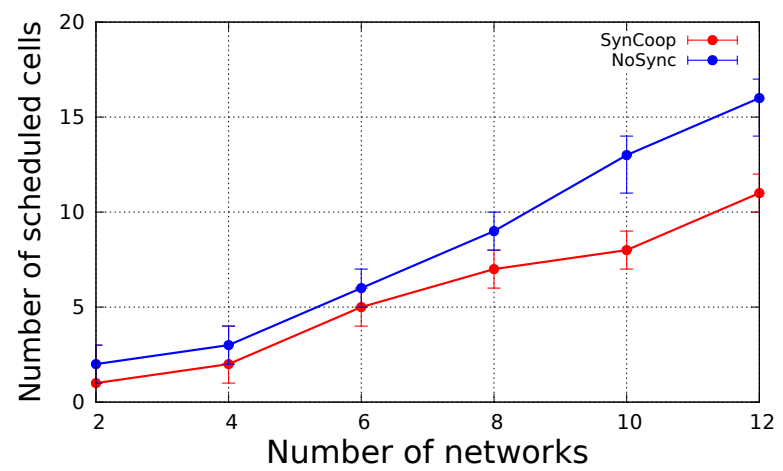

(b) Number of scheduled cells vs. Number of networks

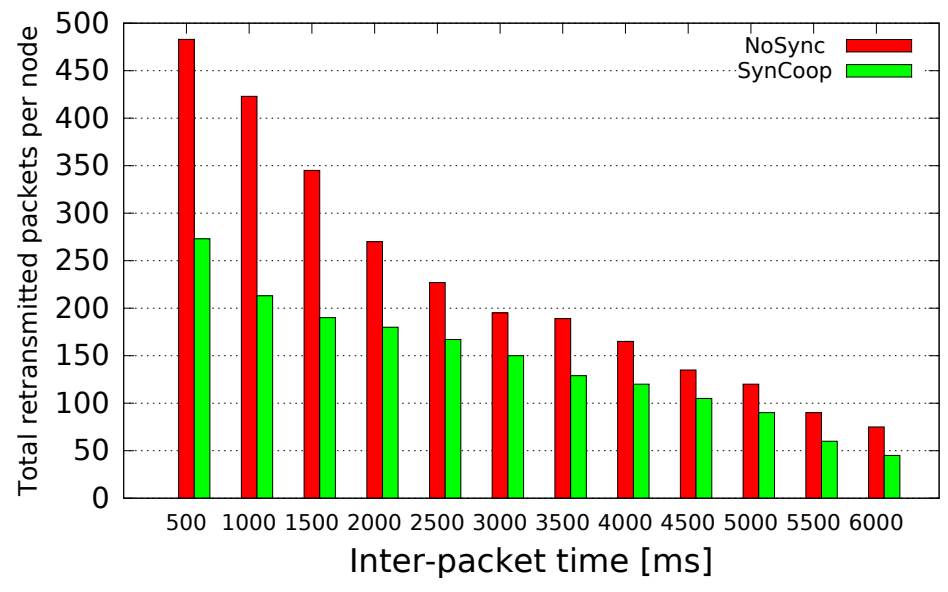

(c) Total number of retransmissions

Figure 15: Efficiency Evaluation -four co-located networks and two nodes per border router

\section{Conclusion and Future Work}

We focused in this paper on very dense deployments of concurrent networks: they compete with the same radio resource, and may lead to collisions. Slow Channel Hopping techniques help to reduce the amount of collisions. However, we experimentally demonstrate that this approach does not reduce the level of interference among co-located TSCH networks. If all the TSCH instances behave independently, the number of collisions quickly increases, having a very negative impact on the end-to-end reliability. We propose consequently to use multiple numbers for the slotframe length, and to synchronize the different border routers. We rely on the Enhanced Beacons transmitted by each border router to re-align the slotframes: a global synchronization is useless, and its is sufficient to align the slot boundaries. By readjusting the clock just before transmitting the EB, and keeping the other children synchronized, even in the worst case, we are consequently able to re-synchronize the different networks without any additional packets. We can even deal with interfering border routers not able to decode their EB, and which need to be synchronized through a chain of other, intermediary border routers. We reduce significantly the number of collisions, providing an high layer-3 reliability (>99\%) even with 6 colocated networks.

In a future work, we aim to explore how to improve the security by enciphering only the sensitive data in the Enhanced Beacons. This way, we could keep on relying on the EB for re-aligning the slotframes while preserving the privacy and the confidentiality. WE also aim at studying the co-existence with other radio technologies using the same unlicensed band: is the slow channel hopping strategy sufficient to combat external interference, or should we propose more specific and efficient solutions? Finally, we aim to explore how multiple TSCH instances may use slotframe lengths which are multiple of each other to make the 


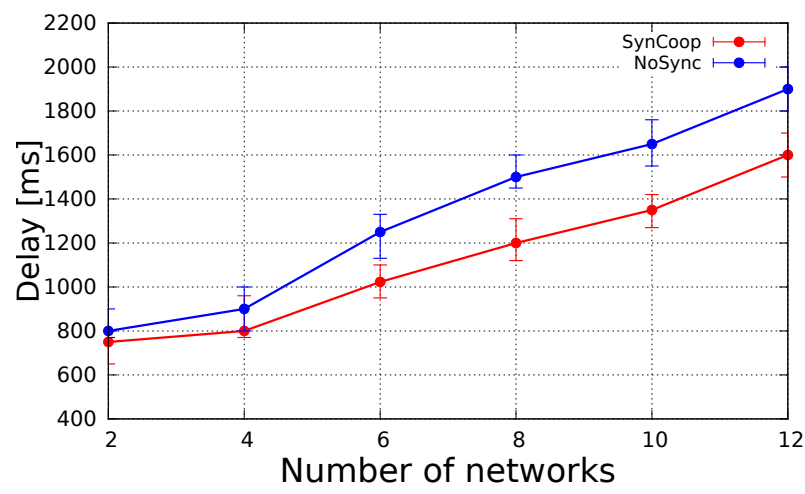

Figure 16: End to end delay vs. Number of networks

collisions repetitive, and easier to solve.

\section{References}

\section{References}

[1] J. Wan, M. Chen, F. Xia, D. Li, K. Zhou, From machine-to-machine communications towards cyber-physical systems, Computer Science and Information Systems 10 (3) (2013) 1105-1128. doi:10.2298/CSIS120326018W

[2] A. Zanella, N. Bui, A. Castellani, L. Vangelista, M. Zorzi, Internet of things for smart cities, IEEE Internet of Things Journal 1 (1) (2014) 22-32. doi:10.1109/JI0T.2014.2306328.

[3] L. D. Xu, W. He, S. Li, Internet of things in industries: A survey, IEEE Transactions on Industrial Informatics 10 (4) (2014) 2233-2243. doi:10.1109/TII.2014.2300753

[4] M. S. Hossain, G. Muhammad, Cloud-assisted industrial internet of things (iiot) - enabled framework for health monitoring, Computer Networks 101 (2016) 192 - 202. doi:10.1016/j.comnet.2016.01.009

[5] F. Shrouf, J. Ordieres, G. Miragliotta, Smart factories in industry 4.0: A review of the concept and of energy management approached in production based on the internet of things paradigm, in: International Conference on Industrial Engineering and Engineering Management, IEEE, 2014, pp. 697-701. doi:10.1109/IEEM.2014.7058728

[6] D. Zhang, J. Wan, C.-H. R. Hsu, A. Rayes, Industrial technologies and applications for the internet of things, Computer

Networks 101 (2016) 1 - 4, industrial Technologies and Applications for the Internet of Things. doi:10.1016/j.comnet. 2016.02 .019

[7] P. Huang, L. Xiao, S. Soltani, M. W. Mutka, N. Xi, The evolution of mac protocols in wireless sensor networks: A survey, IEEE Communications Surveys Tutorials 15 (1) (2013) 101-120. doi:10.1109/SURV.2012.040412.00105.

[8] IEEE Standard for Low-Rate Wireless Personal Area Networks (LR-WPANs), IEEE Std 802.15.4-2015 (Revision of IEEE Std 802.15.4-2011) (April 2016).

[9] ISA-100.11a-2011:, Wireless systems for industrial automation:process control and related applications, International Society of Automation (ISA) Std. 1.

[10] Specification, WirelessHART, 75: TDMA Data-Link Layer, HART Communication Foundation Std., Rev 1.

[11] M. Palattella, N. Accettura, X. Vilajosana, T. Watteyne, L. Grieco, G. Boggia, M. Dohler, Standardized protocol stack

1. for the internet of (important) things, IEEE Communications Surveys Tutorials 15 (3) (2013) 1389-1406. doi:10.1109/ SURV.2012.111412.00158

[12] S. Rajendran, S. R. Chelladurai, A. Aravind, An adaptive road traffic regulation with simulation and internet of things,

1. in: Conference on SIGSIM Principles of Advanced Discrete Simulation (SIGSIM-PADS), ACM, 2016, pp. 3-11. doi: 10.1145/2901378.2901406.

[13] R. Tavakoli, M. Nabi, T. Basten, K. Goossens, An experimental study of cross-technology interference in in-vehicle wireless sensor networks, in: Proceedings of the 19th ACM International Conference on Modeling, Analysis and Simulation of Wireless and Mobile Systems, MSWiM '16, ACM, New York, NY, USA, 2016, pp. 195-204. doi :10.1145/2988287.2989141. URL http://doi.acm.org.insis.bib.cnrs.fr/10.1145/2988287.2989141

580 [14] F. Beltran, S. K. Ray, J. A. Gutiérrez, Understanding the current operation and future roles of wireless networks: Coexistence, competition and co-operation in the unlicensed spectrum bands, IEEE Journal on Selected Areas in Communications 34 (11) (2016) 2829-2837. doi:10.1109/JSAC.2016.2614951

[15] R. Cavallari, F. Martelli, R. Rosini, C. Buratti, R. Verdone, A survey on wireless body area networks: Technologies and design challenges, IEEE Communications Surveys Tutorials 16 (3) (2014) 1635-1657. doi:10.1109/SURV.2014.012214. 00007

[16] C. Withanage, R. Ashok, C. Yuen, K. Otto, A comparison of the popular home automation technologies, in: 2014 IEEE Innovative Smart Grid Technologies - Asia (ISGT ASIA), 2014, pp. 600-605. doi:10.1109/ISGT-Asia.2014.6873860 
[17] Y. Yoon, C. Jung, J. Seo, J. Li, J. Kim, S. Jin, N. Silva, K. Han, A fast adaptive frequency hopping scheme mitigating the effect of interference in bluetooth low energy networks, in: Advances in Services Computing Services Computing Conference (APSCC), 2016, pp. 196-204. doi:10.1007/978-3-319-49178-3_15

[18] T. Hayajneh, G. Almashaqbeh, S. Ullah, A. V. Vasilakos, A survey of wireless technologies coexistence in WBAN: analysis and open research issues, Wireless Networks 20 (8) (2014) 2165-2199. doi:10.1007/s11276-014-0736-8

[19] M. Petrova, J. Riihijarvi, P. Mahonen, S. Labella, Performance study of ieee 802.15.4 using measurements and simulations, in: WCNC, Vol. 1, IEEE, 2006, pp. 487-492. doi:10.1109/WCNC.2006.1683512

[20] L. Angrisani, M. Bertocco, D. Fortin, A. Sona, Experimental study of coexistence issues between ieee 802.11b and ieee 802.15.4 wireless networks, Instrumentation and Measurement, IEEE Transactions on 57 (8) (2008) 1514-1523. doi: 10.1109/TIM.2008.925346.

[21] C.-J. M. Liang, N. B. Priyantha, J. Liu, A. Terzis, Surviving wi-fi interference in low power zigbee networks, in: Conference on Embedded Networked Sensor Systems (SenSys), ACM, 2010, pp. 309-322. doi:10.1145/1869983.1870014

[22] J. H. Park, C. M. Park, S. Lee, B. S. Kim, Interference Resolution Method for IEEE802.15.4-Based Wireless Sensor

Networks, in: Vehicular Technology Conference (VTC Spring), IEEE, 2014, pp. 1-4. doi:10.1109/VTCSpring.2014. 7022954

[23] R. Natarajan, P. Zand, M. Nabi, Analysis of coexistence between ieee 802.15.4, ble and ieee 802.11 in the 2.4 ghz ism band, in: IECON 2016 - 42nd Annual Conference of the IEEE Industrial Electronics Society, 2016, pp. 6025-6032. doi:10.1109/IECON.2016.7793984

[24] F. Hermans, O. Rensfelt, T. Voigt, E. Ngai, L.-A. Norden, P. Gunningberg, SoNIC: Classifying Interference in 802.15.4 Sensor Networks, in: International Conference on Information Processing in Sensor Networks (IPSN), ACM, 2013, pp. 55-66. doi:10.1145/2461381.2461392

[25] R. Ma, H. H. Chen, W. Meng, Dynamic spectrum sharing for the coexistence of smart utility networks and wlans in smart grid communications, IEEE Network 31 (1) (2017) 88-96. doi:10.1109/MNET.2016.1500262NM

[26] M. Gürsu, M. Vilgelm, S. Zoppi, W. Kellerer, Reliable co-existence of 802.15.4e tsch-based wsn and wi-fi in an aircraft cabin, in: International Conference on Communications Workshops (ICC), IEEE, 2016, pp. 663-668. doi:10.1109/ICCW. 2016.7503863

[27] V. Kotsiou, G. Z. Papadopoulos, P. Chatzimisios, F. Theoleyre, Is local blacklisting relevant in slow channel hopping low-power wireless networks?, in: International Conference in Communications (ICC), IEEE, 2017.

[28] N. Abdeddaim, F. Theoleyre, M. Heusse, A. Duda, Adaptive ieee 802.15.4 mac for throughput and energy optimization, in: DCOSS, IEEE, 2013, pp. 223-230. doi:10.1109/DCOSS.2013.44

[29] Y. Iraqi, T. e. Rachidi, P. Y. Kong, Prevention of collisions among two wireless personal area networks, in: Wireless and Mobile Networking Conference (WMNC), IFIP, 2014, pp. 1-7. doi:10.1109/WMNC.2014.6878856

[30] W. Sun, Y. Ge, W.-C. Wong, A lightweight inter-user interference mitigation method in body sensor networks, in: International Conference on Wireless and Mobile Computing, Networking and Communications (WiMob), IEEE, 2012, pp. 34-40. doi:10.1109/WiMOB.2012.6379098

[31] L. H. Correia, T.-D. Tran, V. N. Pereira, J. C. Giacomin, J. M. S. Silva, DynMAC: A resistant MAC protocol to coexistence in wireless sensor networks, Computer Networks 76 (2015) $1-16$. doi:10.1016/j.comnet.2014.10.019

625 [32] P.-Y. Kong, A distributed mac scheme to avoid collisions among multiple wireless personal area networks, in: Wireless Communications and Networking Conference (WCNC), IEEE, 2013, pp. 71-76. doi:10.1109/WCNC.2013.6554541

[33] N. Torabi, K. Rostamzadeh, V. C. M. Leung, Ieee 802.15.4 beaconing strategy and the coexistence problem in ism band, IEEE Transactions on Smart Grid 6 (3) (2015) 1463-1472. doi:10.1109/TSG.2014.2344038

[34] L. M. Feeney, V. Fodor, Reliability in co-located 802.15.4 personal area networks, in: International Workshop on Pervasive Wireless Healthcare (MobiHealth), ACM, 2016, pp. 5-10. doi:10.1145/2944921.2944923

[35] M. Alasti, M. Barbi, K. Sayrafian, Uncoordinated strategies for inter-ban interference mitigation, in: International Symposium on Personal, Indoor, and Mobile Radio Communication (PIMRC), IEEE, 2014, pp. 2150-2154. doi: 10.1109/PIMRC.2014.7136528.

[36] X. Vilajosana, K. Pister, T. Watteyne, Minimal 6tisch configuration, draft-ietf-6tisch-minimal-21, IETF (February 2017).

635 [37] Ipv6 over the tsch mode of ieee 802.15.4e, https://datatracker.ietf.org/wg/6tisch

[38] J. Eidson, K. Lee, Ieee 1588 standard for a precision clock synchronization protocol for networked measurement and control systems, in: Sensors for Industry Conference, ISA/IEEE, 2002, pp. 98-105. doi:10.1109/SFICON.2002.1159815.

[39] S. Ganeriwal, R. Kumar, M. B. Srivastava, Timing-sync protocol for sensor networks, in: international conference on Embedded networked sensor systems (SenSys), ACM, 2003, pp. 138-149. doi:10.1145/958491.958508

640 [40] K. Pister, L. Doherty, Tsmp: Time synchronized mesh protocol, IASTED Distributed Sensor Networks (2008) $391-398$.

[41] T. Chang, T. Watteyne, K. Pister, Q. Wang, Adaptive synchronization in multi-hop TSCH networks, Computer Networks 76 (2015) 165 - 176. doi:dx.doi.org/10.1016/j.comnet.2014.11.003

[42] D. Stanislowski, X. Vilajosana, Q. Wang, T. Watteyne, K. S. J. Pister, Adaptive synchronization in ieee802.15.4e networks, IEEE Transactions on Industrial Informatics 10 (1) (2014) 795-802. doi:10.1109/TII.2013.2255062.

645 [43] A. Carroll, G. Heiser, An analysis of power consumption in a smartphone, in: Annual Technical Conference Conference on USENIX (USENIXATC), 2010, pp. 21-21.

[44] F. Theoleyre, G. Papadopoulos, Experimental Validation of a Distributed Self-Configured 6TiSCH with Traffic Isolation in Low Power Lossy Networks, in: International Conference on Modeling, Analysis and Simulation of Wireless and Mobile Systems (MSWiM), ACM, 2016, pp. 102-110. doi:10.1145/2988287.2989133.

650 [45] T. Schmid, Z. Charbiwala, R. Shea, M. B. Srivastava, Temperature compensated time synchronization, IEEE Embedded Systems Letters 1 (2) (2009) 37-41. doi:10.1109/LES.2009.2028103.

[46] T. Watteyne, M. R. Palattella, L. A. Grieco, Using IEEE 802.15.4e Time-Slotted Channel Hopping (TSCH) in the Internet 
of Things (IoT): Problem Statement, rfc 7554, IETF (Oct. 2015). doi:10.17487/rfc7554

[47] J. Klinglmayr, C. Bettstetter, Self-organizing synchronization with inhibitory-coupled oscillators: Convergence and robustness, ACM Trans. Auton. Adapt. Syst. 7 (3) (2012) 30:1-30:23. doi:10.1145/2348832.2348833

[48] Q. Li, D. Rus, Global clock synchronization in sensor networks, IEEE Transactions on computers 55 (2) (2006) $214-226$. doi:10.1109/TC.2006.25

[49] E. D. Brooks, The butterfly barrier, International Journal of Parallel Programming 15 (4) (1986) 295-307. doi:10.1007/ BF01407877 